\title{
Decrease in the Roughness of Vapor-Deposited Gold Surfaces Induced by Surface Mobility ${ }^{\dagger}$
}

\author{
J. L. Zubimendi, ${ }^{\ddagger}$ M. E. Vela, ${ }^{\ddagger}$ R. C. Salvarezza, ${ }^{\ddagger}$ L. Vázquez, ${ }^{\S}$ J. M. Vara,,${ }^{\prime}$ and \\ A. J. Arvia*,‡
}

\begin{abstract}
Instituto de Investigaciones Fisicoquímicas Teóricas y Aplicadas (INIFTA), Casilla de Correo 16. Sucursal 4, 1900 La Plata, Argentina, Instituto de Ciencia de Materiales, Consejo Superior de Investigaciones Científicas, Departamento de Fisica Aplicada C-XII, Universidad Autónoma de Madrid, 28049 Madrid, Spain, and Instituto de Ciencia de Materiales, Consejo Superior de Investigaciones Científicas, Departamento de Química Física Aplicada C-II, Universidad
\end{abstract} Autónoma de Madrid, 28049 Madrid, Spain

Received September 1, 1994. In Final Form: December 5, $1994^{\otimes}$

\begin{abstract}
The evolution of the roughness exponent of vapor-deposited thin gold films (the object) on smooth glass substrate at $298 \mathrm{~K}$ has been observed by scanning tunneling microscopy. This evolution was enhanced by keeping the films at $323 \mathrm{~K}$ in contact with aqueous $0.5 \mathrm{M} \mathrm{H}_{2} \mathrm{SO}_{4}$ containing different $\mathrm{KCl}$ concentrations for $24 \mathrm{~h}$. It implies a decay of the surface roughness for lengths larger than the grain size of the object leading to better ordered surface structures. Results are compared to those obtained during film grown by increasing the temperature of the glass substrate from 298 to $673 \mathrm{~K}$. The analysis of surface roughness data indicated an enhancement of surface atom diffusion in ordering the gold surface structure, by either anion adsorption or deposition temperature.
\end{abstract}

\section{Introduction}

Surface roughness reflects physical processes operating on solid surfaces during their growth and evolution toward the equilibrium form. To understand the dynamics of growing interfaces, ${ }^{1}$ both continuum and atomistic models have been proposed. The main physical processes used for modeling roughness are intrinsic noise in surface growth, surface tension, and surface atom diffusion. ${ }^{2}$ When the surface dynamics depends only on local processes, objects with a self-affine fractal surface with compact nonfractal mass are formed. ${ }^{1}$ In this case, $\xi$, the interface width, scales with $L_{\mathrm{s}}$, the substrate size, according to $\xi \propto$ $\mathrm{L}_{s}{ }^{\alpha}$, where $\alpha$ is the characteristic surface roughness exponent which becomes the relevant quantity characterizing the surface properties. Thus, values of $\alpha$ ranging between 0 and 1 are expected for 3D systems according to the three above mentioned physical contributions to surface dynamics. ${ }^{3}$

Recently, data derived from scanning tunneling microscopy (STM) imaging ${ }^{4-6}$ were used for the fractal characterization of gold film surfaces prepared by vapor deposition on a smooth glass substrate at the growth rate $V=30 \mathrm{~nm} \mathrm{~s}^{-1}$, and substrate temperature $T_{\mathrm{s}}=298 \mathrm{~K}$. Then it was concluded that the surface of these films

${ }^{\dagger}$ Presented at the symposium on Advances in the Measurement and Modeling of Surface Phenomena, San Luis, Argentina, August 24-30, 1994.

‡INIFTA.

\$ Departamento de Física Aplicada C-XII.

" Departamento de Química Física Aplicada C-II.

${ }^{\otimes}$ Abstract published in Advance ACS Abstracts, January 1, 1996.

(1) Family, F. Physica A 1990, 168, 561, and references therein.

(2) Villain, J. J. Phys. I 1991, 1, 19.

(3) Vvdensky, D. D.; Zangwill, A.; Luse, C. N.; Wilby, M. R. Phys. Rev. E 1993, 48, 852.

(4) Salvarezza, R. C.; Vázquez, L.; Herrasti, P.; Ocón, P.; Vara, J. M.; Arvia, A. J. Europhys. Lett. 1992, 20, 727.

(5) Vázquez, L.; Salvarezza, R. C.; Herrasti, P.; Ocón, P.; Vara, J. M.; Arvia, A. J. Appl. Surf. Sci. 1993, 70-71, 413.

(6) Herrasti, P.; Ocón, P.; Vázquez, L.; Salvarezza, R. C.; Vara, J. M.; Arvia, A. J. Phys. Rev. A 1990, 45, 7440. behaves as a self-affine fractal with roughness exponents, $\alpha(\mathrm{I}) \cong 0.90$ for $L_{\mathrm{s}}<d_{\mathrm{s}}$, and $\alpha(\mathrm{II}) \cong 0.36$ for $L_{\mathrm{s}}>d_{\mathrm{s}}$, where $d_{\mathrm{s}}$ is the average columnar size. ${ }^{4-6}$ Although the value $\alpha(\mathrm{II}) \cong 0.36$ appears to be consistent with the predictions of the stochastic Kardar, Parisi, and Zhang (KPZ) model, ${ }^{7}$ this theory does not incorporate the effect of grain formation. Thus, in principle, this coincidence appears to be fortuitous. On the other hand, the value $\alpha(\mathrm{I}) \cong 0.9$ for $L_{\mathrm{s}}<d_{\mathrm{s}}$, indicates a smooth textured columnar surface which can be produced by the surface mobility of gold atoms. Smoothening leads to the disappearance of irregularities within a diffusion length close to $d_{\mathrm{s}}{ }^{4-6}$ In this case, the value of $\alpha(\mathrm{I}) \cong 0.9$ exceeds the value of $\alpha$ expected from continuum models incorporating surface relaxation, ${ }^{8}$ although that figure is consistent with values of $\alpha$ derived from simple growth models in which particles are randomly deposited onto a substrate and relax to kink sites maximizing the number of saturated bonds. ${ }^{9,10}$ Accordingly, it appears that surface atom diffusion changes the surface properties of deposited films from fractal to nonfractal.

A considerable increase in surface metal atom mobility can be produced during film formation by increasing the temperature of the substrate or by the adsorption of foreign particles. A strong surface rearrangement has been observed for electrodeposited metal films in contact with aqueous solutions containing adsorbable anions. ${ }^{11}$

In this work the surface atom diffusion contribution to surface roughness development is analyzed during the growth of thin gold films by changing $T_{\mathrm{s}}$, the substrate temperature, and for postdeposition surface roughness evolution by the adsorption of specific solution constituents. The disappearance of the columnar structure with $\alpha \cong 0.4$ and the appearance of faceted surface structures imply values of changing gradually from $\alpha \cong 0.4$ upward as the contribution of surface atom diffusion increases.

(7) Kardar, M.; Parisi, G.; Zhang, Y. C. Phys. Rev. Lett. 1986, 56, 889.

(8) Lai, Z. W.; Das Sarma, S. Phys. Rev. Lett. 1991, 66, 2348.

(9) Das Sarma, S.; Tamborenea, P. Phys. Rev. Lett. 1991, 66, 325.

(10) Wolf, D.; Villain, J. Europhys. Lett. 1990, 13, 389.

(11) Alonso, C.; Salvarezza, R. C.; Vara, J. M.; Arvia, A. J. Electrochim. Acta 1990, 35, 1331 . 


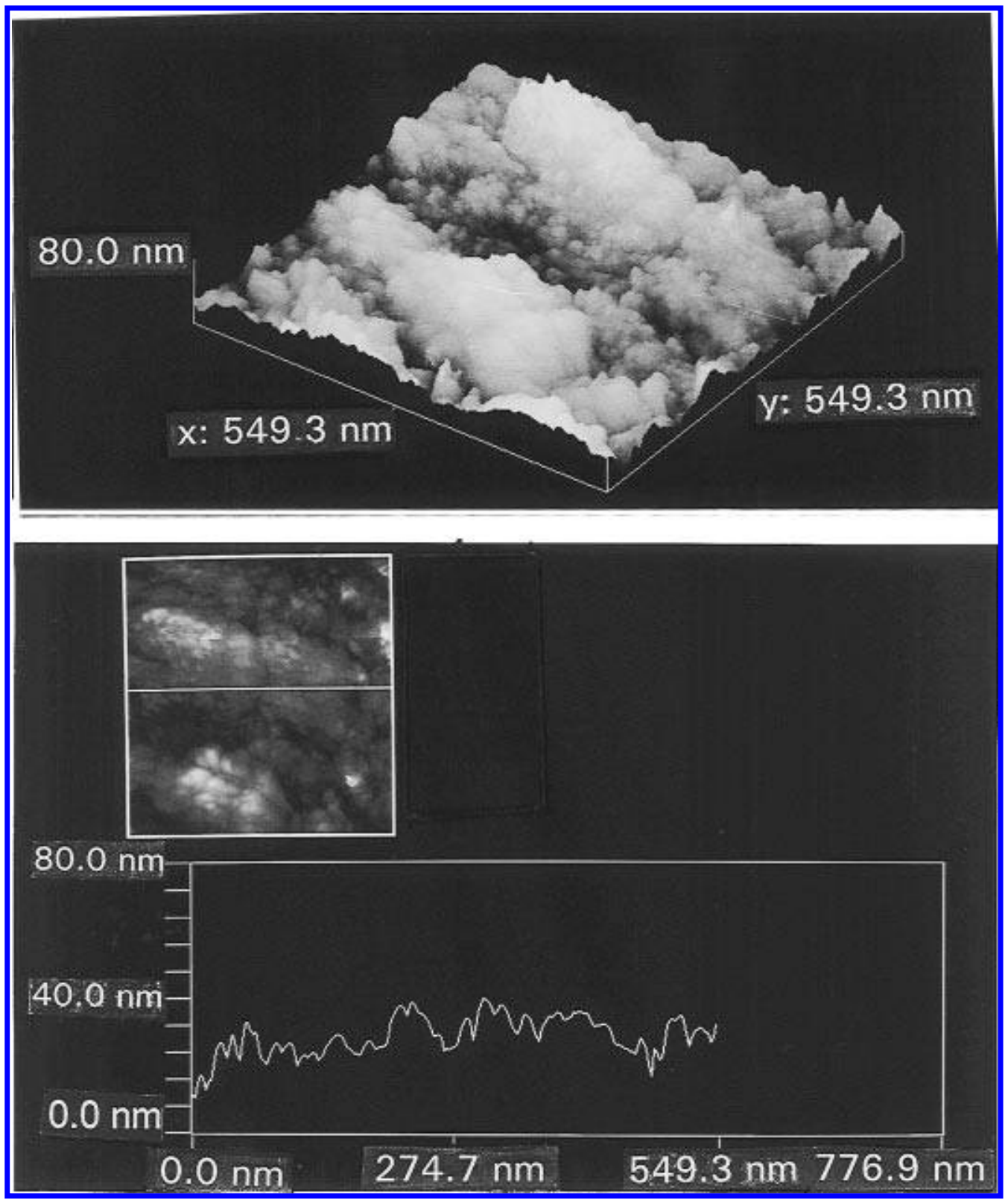

Figure 1. STM image of a vapor-deposited gold film grown at $v=0.1 \mathrm{~nm} \mathrm{~s}^{-1}$ and $T_{\mathrm{s}}=298 \mathrm{~K}$ : (a, top) three-dimensional image; (b, bottom) cross section of the image shown in part a.

\section{Experimental Section}

Gold deposits were grown in an evaporator chamber on smooth glass substrates previously cleaned in an ultrasonic bath by sequentially using water, trichloroethylene, acetone, and ethanol. ${ }^{4-6}$ The maximum deviation of the incident particles from the substrate normal was $9^{\circ}$. The experimental conditions at the evaporator chamber were as follows: pressure $P=10^{-4}$ Torr (air as residual gas), average deposit growth rate $\langle v\rangle=0.1$ $\mathrm{nm} \mathrm{s}^{-1}$, and $298 \mathrm{~K}<T_{\mathrm{s}}<673 \mathrm{~K}$. The average film thickness was $\langle h\rangle \cong 200 \mathrm{~nm}$ as has been estimated by the weight method. Previous results for this system ${ }^{5}$ showed that for $\langle h\rangle \geq 170 \mathrm{~nm}$, the root mean square roughness of the deposit surface approached a steady state. ${ }^{5}$

A number of gold films grown at $298 \mathrm{~K}$ were kept immersed in aqueous $0.5 \mathrm{M} \mathrm{H}_{2} \mathrm{SO}_{4}+x \mathrm{M} \mathrm{KCl}, 0 \leq x \leq 0.5 \mathrm{M}$, at $323 \mathrm{~K}$ under purified nitrogen saturation for 24 -h periods to observe the influence of the electrolyte composition on roughness relaxation. The experimental conditions were selected on the basis of previous results on electrodeposited gold films. ${ }^{11}$

A McAllister STM operating in air was used to investigate the surface morphology of gold deposits. The STM was calibrated by imaging highly oriented pyrolytic graphite (HOPG). Tips were made from $0.25 \mathrm{~mm}$ diameter platinum-iridium wires directly by cutting. Although different tips were used to minimize tip geometric artifacts, no influence of the tip shape on our experimental data was found. STM measurements were made using a $0.05-\mathrm{V}$ bias voltage with the tip $(+)$ at $1-2 \mathrm{nA}$ constant current. Data were acquired in a fully automated worksation and stored as digitized images with $200 \times 200$ pixels. Occasionally, images with $400 \times 400$ pixels were taken with similar results.

Usually, STM measurements involve the uncertainty as to whether the tip scans over a parallel or a tilted plane of the sample. The presence of a tilted plane introduces an overestimation in the value of $\alpha$ as resulted from computer-generated surfaces with added planes. STM plane removal options in the software have been successfully used to remove the tilted plane. ${ }^{12}$ Therefore, STM data were analyzed after the instrument plane fitting and substracting procedure. ${ }^{6}$

\section{Results}

STM images of vapor-deposited gold film surfaces resulting from those runs made at $V=0.1 \mathrm{~nm} \mathrm{~s}^{-1}$ and $T_{\mathrm{s}}$

(12) Krim, J.; Hevaert, I.; Haesendock, C.; Bruynseraede, Y. Phys. Rev. Lett. 1990, 70, 57. 


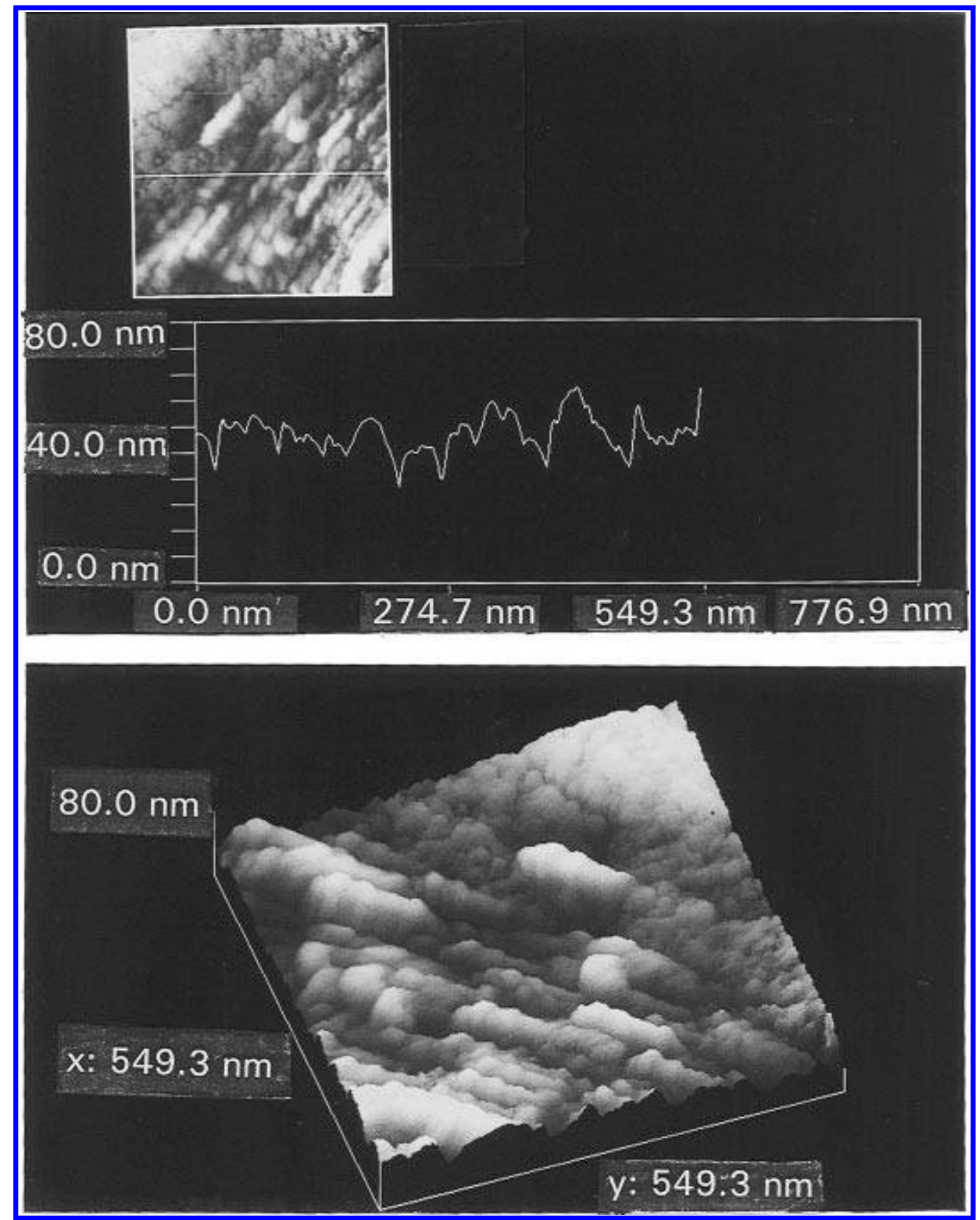

Figure 2. STM images of a vapor-deposited gold film grown at $v=0.1 \mathrm{~nm} \mathrm{~s}^{-1}$ and $T_{\mathrm{s}}=298 \mathrm{~K}$ after the film was kept in contact with $0.5 \mathrm{M} \mathrm{H}_{2} \mathrm{SO}_{4}$ for $24 \mathrm{~h}$ at $323 \mathrm{~K}$ : (a, top) cross section of an image $549 \times 549 \mathrm{~nm}^{2}$; (b, bottom) three-dimensional view of the same image.

$=298 \mathrm{~K}$ (Figure 1a) show rounded top columns with $\left\langle d_{\mathrm{s}}\right\rangle$ $\cong 20 \mathrm{~nm}$, branched voids, and small columns which agglomerate in larger rounded elements. The cross sections of these STM images (Figure 1b) correspond to jagged profiles ${ }^{13}$ due to the height fluctuation of columnar elements. ${ }^{4-6}$ These fluctuations arise from shadowing between neighboring growing columns. ${ }^{4}$ As already reported, small rounded top column surfaces are rather smooth. ${ }^{4}$

On the other hand, after immersion in $0.5 \mathrm{M} \mathrm{H}_{2} \mathrm{SO}_{4}$ at $323 \mathrm{~K}$ for $24 \mathrm{~h}$, the topography of gold films undergoes some changes as columns are aligned following preferred directions (Figure 2a). However, cross sections of these STM images (Figure 2b) show surface profiles very similar to those recorded before immersion in $0.5 \mathrm{M} \mathrm{H}_{2} \mathrm{SO}_{4}$ at 323

(13) Krim, J.; Indekeu, J. O. Phys. Rev. E 1993, 48, 1576.
$\mathrm{K}$ for $24 \mathrm{~h}$. After immersion in $0.5 \mathrm{M} \mathrm{H}_{2} \mathrm{SO}_{4}+0.01 \mathrm{M} \mathrm{KCl}$ at $323 \mathrm{~K}$ for $24 \mathrm{~h}$, the rounded shaped contour of columns tends to disappear and geometric crystals dominate the surface topography (Figure 3). This effect become more evident by increasing the $\mathrm{KCl}$ concentration in the solution. For instance, after immersion in aqueous $0.5 \mathrm{M}$ $\mathrm{H}_{2} \mathrm{SO}_{4}+0.5 \mathrm{M} \mathrm{KCl}$ at $323 \mathrm{~K}$ for $24 \mathrm{~h}$ (Figure $4 \mathrm{a}$ ), the columnar structure of the gold film is completely suppressed and replaced by aligned geometric crystals following well-defined directions which dominate the surface topography. These crystals are 15-20 nm in average size, and their cross section, as derived from STM images (Figure 4b), shows profiles with a smoother texture in relation to those observed for gold films before their immersion in $\mathrm{KCl}$-containing solutions.

Finally, for gold films prepared at $v=0.1 \mathrm{~nm} \mathrm{~s}^{-1}$ and $T_{\mathrm{s}}=673 \mathrm{~K}$, STM images also show faceted surfaces (Figure $5 a)$ with a texture smoother (Figure $5 b$ ) than that of other 


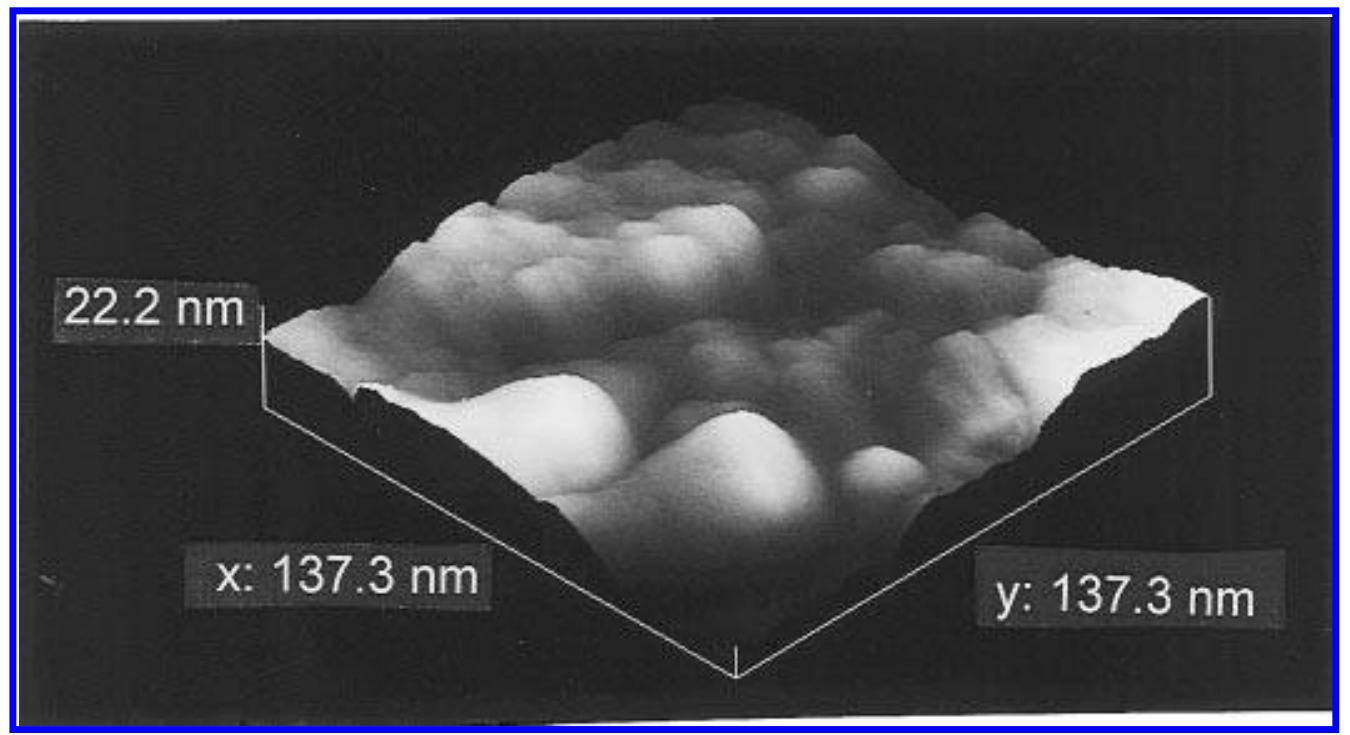

Figure 3. Three-dimensional STM image of a vapor-deposited gold film grown at $v=0.1 \mathrm{~nm} \mathrm{~s}^{-1}$ and $T_{\mathrm{s}}=298 \mathrm{~K}$ after the film was kept in contact with $0.5 \mathrm{M} \mathrm{H}_{2} \mathrm{SO}_{4}+0.01 \mathrm{M} \mathrm{KCl}$ for $24 \mathrm{~h}$ at $323 \mathrm{~K}$.

gold films studied in this work. In this case, the crystal size largely exceeds the size of those grown at $T_{\mathrm{s}}=298$ $\mathrm{K}$.

\section{Discussion}

The analysis of STM imaging data resulting from gold film surfaces was based on the dynamic scaling method. ${ }^{1,4-6}$ Accordingly, $\xi_{\text {stm }}$, the root mean square roughness measured by STM on STM scans of length $L_{\mathrm{s}}$, scales as

$$
\xi_{\mathrm{stm}}\left(L_{\mathrm{s}}, h\right) \propto L_{\mathrm{s}}^{\alpha} f(x)
$$

where $f(x)=\langle h\rangle / \mathrm{L}^{\gamma}, f(x)=$ constant for $x \Rightarrow \infty$, and $f(x)=$ $x^{\alpha / \gamma}$ for $\boldsymbol{x} \Rightarrow 0$, and $\xi$ is defined as

$$
\xi_{\mathrm{stm}}\left(L_{\mathrm{s}}\right)=\left[(1 / N) \sum\left[h\left(x_{i}\right)-\langle h\rangle\right]^{2}\right]^{1 / 2}
$$

where $h\left(x_{i}\right)$ is the deposit height measured along the $x$-direction at the position $x_{i}$, and $\langle h\rangle$ is the average height of the sample formed by $N$ points. After a certain critical time or thickness is reached, eq 1 becomes

$$
\xi_{\mathrm{stm}}\left(L_{\mathrm{s}}\right) \propto L_{\mathrm{s}}^{\alpha}
$$

According to eq 3 the value of $\alpha$ can be obtained from a $\log \xi_{\text {stm }}$ vs $\log L_{\mathrm{s}}$ plot. For $T_{\mathrm{s}}=298 \mathrm{~K}, \log \xi_{\mathrm{stm}}$ vs $\log L_{\mathrm{s}}$ plots exhibit two straight line portions with a crossing point at $\log L_{\mathrm{s}}=1.7$ and a saturation region close to the image size (Figure 6a). The slopes of the straight lines are $\alpha(\mathrm{I})=0.77 \pm 0.07$ for $\log L_{\mathrm{s}}<1.7$, and $\alpha(\mathrm{II})=0.37$ \pm 0.06 for $\log L_{\mathrm{s}}>1.7$. It should be noted that for computer-simulated fractals, data covering 4 to 5 orders of magnitude are required for logarithmic fitting, but for experimental systems, this goal is less ambitious due to specific limitations. Then, $\log \xi_{\mathrm{stm}}$ vs $\log L_{\mathrm{s}}$ linear plots covering at least 1 order of magnitude or thereabouts are considered acceptable from experimental data.

As already reported, ${ }^{5}$ the capability of the dynamic scaling method for rough surface analysis was tested for several computer-generated self-affine fractal surfaces with preset values of $\alpha_{\mathrm{th}}$, built on $200 \times 200$ and $256 \times$ 256 square grids, using the successive random addition algorithm. ${ }^{14}$ A good agreement between $\alpha_{\mathrm{m}}$, the measured value of $\alpha$, and $\alpha_{\mathrm{th}}$ was found in the $0.2<\alpha_{\mathrm{th}}<0.6$ range, whereas for $\alpha_{\mathrm{th}}>0.6, \alpha_{\mathrm{m}}$ became smaller than $\alpha_{\mathrm{th}}$. A better agreement with the data can be obtained by increasing substantially the number of points in the square grid, for instance, to $1000 \times 1000$ pixels. However, the $200 \times 200$ grid involves a short STM imaging time minimizing drift effects. Thus, from the compromise between grid size and image drift, it is advisable to select a small grid to minimize drift effects and use the $\alpha_{\mathrm{m}}$ vs $\alpha_{\text {th }}$ plot to make a correction to the $\alpha$ values measured from STM images. Then, after correction $\langle\alpha(\mathrm{I})\rangle$ and $\langle\alpha$ (II) $\rangle$, the averaged values of $\alpha$ (I) and $\alpha$ (II) resulting from 20 different STM images taken in the $20 \mathrm{~nm}<L_{\mathrm{s}}<550$ nm range are $\langle\alpha(\mathrm{I})\rangle=0.92 \pm 0.07$ and $\langle\alpha(\mathrm{II})\rangle=0.40 \pm$ 0.05 . Similarly, for gold deposits grown at $T_{\mathrm{s}}=298 \mathrm{~K}$ and kept in contact with $0.5 \mathrm{M} \mathrm{H}_{2} \mathrm{SO}_{4}$ at $323 \mathrm{~K}$, the $\log \xi_{\text {stm }}$ vs $\log L_{\mathrm{s}}$ plots yielded after correction $\langle\alpha(\mathrm{I})\rangle=0.90 \pm 0.05$ and $\langle\alpha($ II $)\rangle=0.43 \pm 0.04$.

For gold deposits grown at $T_{\mathrm{s}}=298 \mathrm{~K}$ after being maintained in contact with $0.5 \mathrm{M} \mathrm{H}_{2} \mathrm{SO}_{4}+0.01 \mathrm{M} \mathrm{KCl}$, the $\log \xi_{\mathrm{stm}}$ vs $\log L_{\mathrm{s}}$ plots also yielded two straight lines, although in this case after correction it resulted in $\langle\alpha(\mathrm{I})\rangle$ $=0.90$ for $\log L_{\mathrm{s}}<1.5$, and $\langle\alpha(\mathrm{II})\rangle=0.49 \pm 0.06$ for $\log$ $L_{\mathrm{s}}>1.5$. This increase in the value of $\langle\alpha(\mathrm{II})\rangle$ suggests that smoothening of the original topography has occurred due to the presence of $\mathrm{Cl}^{-}$ions in the solution. The effect of these ions as promoters of gold surface smoothening was confirmed by increasing the $\mathrm{KCl}$ concentration in the solution. Thus, for gold deposits grown at $T_{\mathrm{s}}=298 \mathrm{~K}$ and kept in contact with $0.5 \mathrm{M} \mathrm{H}_{2} \mathrm{SO}_{4}+0.5 \mathrm{M} \mathrm{KCl}$ at $323 \mathrm{~K}$ for $24 \mathrm{~h}$, the $\log \xi_{\mathrm{stm}}$ vs $\log L_{\mathrm{s}}$ plot exhibits two straight lines with $\alpha(\mathrm{I})=0.74 \pm 0.07$ for $\log L_{\mathrm{s}}<1.7$, and $\alpha(\mathrm{II}) \cong$ $0.54 \pm 0.05$ for $\log L_{\mathrm{s}}>1.7$ (Figure $6 \mathrm{~b}$ ). In this case, from the analysis of 20 different STM images covering the 20$\mathrm{nm}<L_{\mathrm{s}}<550-\mathrm{nm}$ range, after correction, it results in $\langle\alpha(\mathrm{I})\rangle=0.88 \pm 0.07$ and $\langle\alpha(\mathrm{II})\rangle=0.56 \pm 0.05$.

It should be noted that gold deposits grown at $298 \mathrm{~K}$ and aged in different electrolyte solutions at $323 \mathrm{~K}$ for 24 $\mathrm{h}$ exhibit a crossover point in the $\log \xi_{\mathrm{stm}}$ vs $\log L_{\mathrm{s}}$ plots covering the $20-\mathrm{nm}<L_{\mathrm{s}}<40-\mathrm{nm}$ range, i.e. at $L_{\mathrm{s}}$ values

(14) Voss, R. In Fundamental Algorithms in Computer Graphics; Earnshaw, R. A., Ed.; Springer-Verlag: Berlin, 1985. 

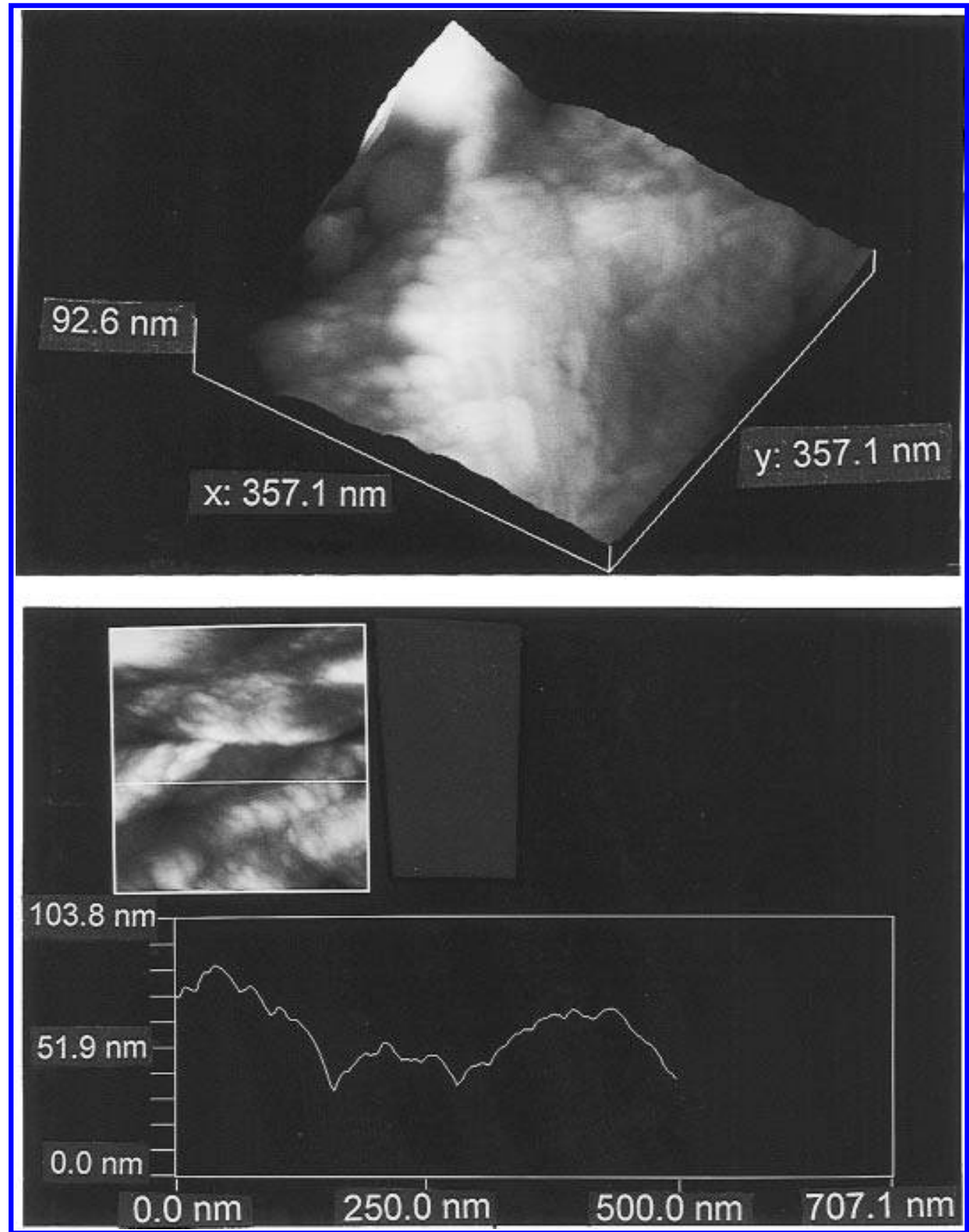

Figure 4. STM images of a vapor-deposited gold film grown at $v=0.1 \mathrm{~nm} \mathrm{~s}^{-1}$ and $T_{\mathrm{s}}=298 \mathrm{~K}$ after the film was kept in contact with $0.5 \mathrm{M} \mathrm{H}_{2} \mathrm{SO}_{4}+0.5 \mathrm{M} \mathrm{KCl}$ for $24 \mathrm{~h}$ at $323 \mathrm{~K}$ : (a, top) three-dimensional image; (b, bottom) cross section of the image shown in part a.

Table 1. Corrected Average Values of $\alpha$ Resulting at Different Electrolyte Composition and $T_{s}$

\begin{tabular}{clccc}
\hline$T_{\mathrm{s}}, \mathrm{K}$ & eletrolyte solution & temperature, $\mathrm{K}$ & $\langle\alpha(\mathrm{I})\rangle$ & $\langle\alpha(\mathrm{II})\rangle$ \\
\hline 298 & none & & $0.92 \pm 0.07$ & $0.40 \pm 0.05$ \\
298 & $\mathrm{H}_{2} \mathrm{SO}_{4}$ & 323 & $0.90 \pm 0.07$ & $0.43 \pm 0.04$ \\
298 & $\mathrm{H}_{2} \mathrm{SO}_{4}$ & 323 & $0.90 \pm 0.07$ & $0.49 \pm 0.06$ \\
& $\quad+0.01 \mathrm{M} \mathrm{KCl}$ & & & \\
298 & $\begin{array}{c}\mathrm{H}_{2} \mathrm{SO}_{4} \\
\quad+0.5 \mathrm{M} \mathrm{KCl} \\
\text { none }\end{array}$ & 323 & $0.88 \pm 0.07$ & $0.56 \pm 0.06$ \\
673 & & $0.93 \pm 0.07$ &
\end{tabular}

which are close to the value of $d_{\mathrm{s}}$. Then, the value $\langle\alpha(\mathrm{I})\rangle$ $\cong 0.9$ obtained for $L_{\mathrm{s}}<20-40 \mathrm{~nm}$ should be assigned to the smooth surface domains of either columns or geometric crystals forming the deposits, whereas values of $\langle\alpha(\mathrm{II})\rangle$, which are always lower than those of $\langle\alpha(\mathrm{I})\rangle$ for $L_{\mathrm{s}}>20-40$ $\mathrm{nm}$, have to be related to height fluctuations involving either columns or crystals.
The interpretation of the physical origin of the two regions in the $\log \xi_{\text {stm }}$ vs $\log L_{\mathrm{s}}$ plots which are revealed by changing the lateral correlation length, i.e. the microscope resolution, can be supported by scaling computer-simulated surfaces resulting from an ensemble of columns fluctuating in height with known values of $\alpha_{s}$. The surface of each column is represented by a smooth rounded cusp of diameter $d_{\mathrm{s}}$. For this surface model the $\log \xi$ vs $\log L$ plots exhibit one or two linear regions depending on the $L / d_{\mathrm{s}}$ ratio. For $L / d_{\mathrm{s}} \gg 1$ only one region with slope $\alpha_{\mathrm{s}}$ is observed. Otherwise, for $L / d_{\mathrm{s}} \leq 20$ two linear regions with $\alpha \Rightarrow 1$ for $L<d_{\mathrm{s}}$ and $\alpha=\alpha_{\mathrm{s}}$ for $L_{\mathrm{s}}>$ $d_{\mathrm{s}}$ are observed. Finally, for $L / d_{\mathrm{s}} \Longrightarrow 1$ only the region with $\alpha \cong 1$ results from the $\log \xi$ vs $\log L$ plots. Note that $\alpha$ $\cong 1$ is consistent with a smooth rounded cusp surface. Thus, from these simulations it can be concluded that the two regions in the $\log \xi$ vs $\log L$ plots correspond to truly different scaling ranges. 


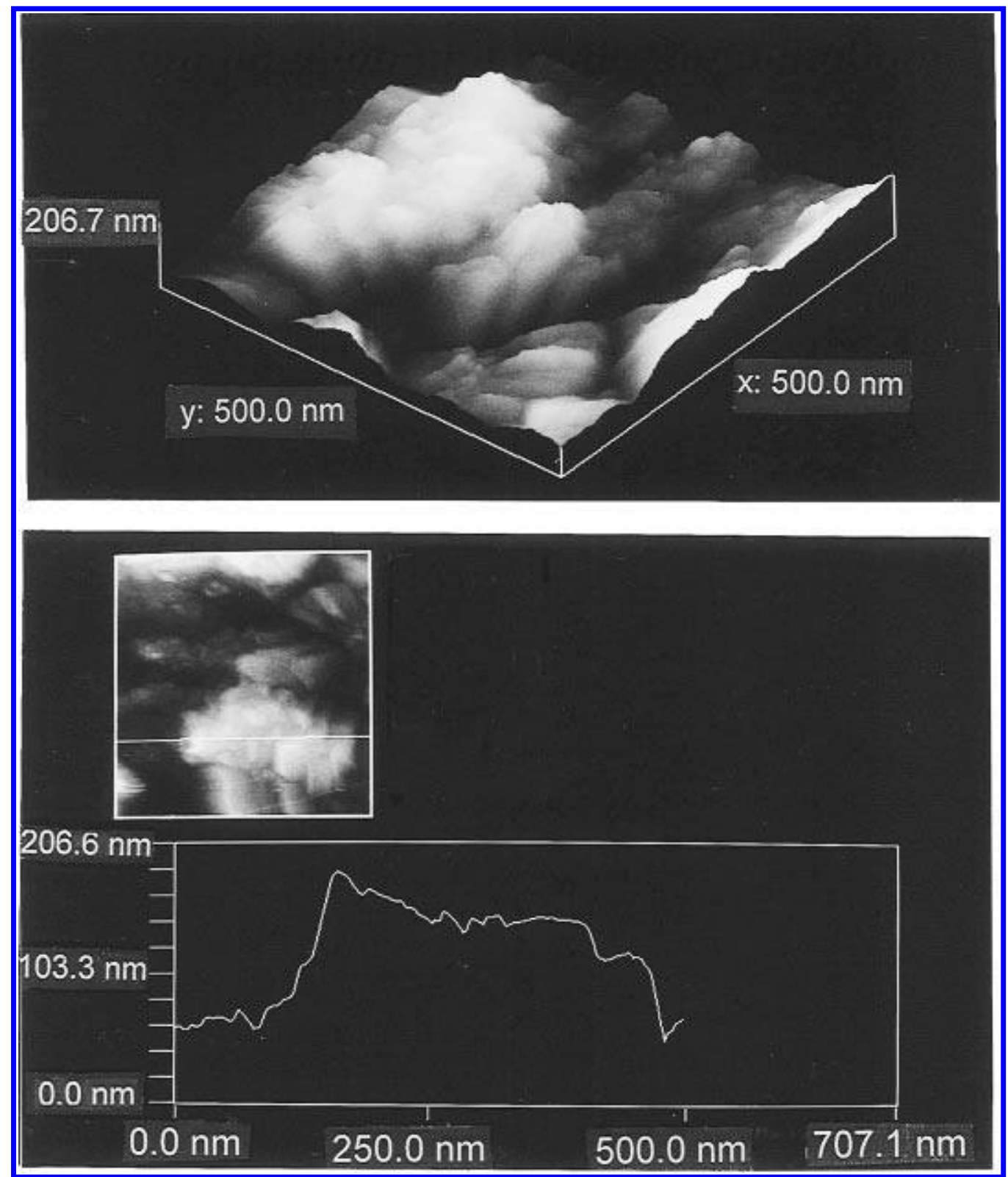

Figure 5. STM images of a vapor-deposited gold film grown at $v=0.1 \mathrm{~nm} \mathrm{~s}^{-1}$ and $T_{\mathrm{s}}=673 \mathrm{~K}$ : (a, top) three-dimensional image; (b, bottom) cross section of the image shown in part $a$.

On the other hand, for gold deposits grown at $v=0.1$ $\mathrm{nm} \mathrm{s}^{-1}$ and $T_{\mathrm{s}}=673 \mathrm{~K}$, i.e. those films which exhibit a predominantly faceted topography, the $\log \xi_{\mathrm{stm}}$ vs $\log L_{\mathrm{s}}$ plots (Figure 6c) lead to a single straight line with $\alpha(\mathrm{I}) \cong$ $0.76 \pm 0.07$ and a small saturation region which appears when $L_{\mathrm{s}}$ is close to the STM image size. Then, from the analysis of $20 \mathrm{STM}$ images for $T_{\mathrm{s}}=673 \mathrm{~K}$, in the $20-\mathrm{nm}$ $<L_{\mathrm{s}}<550$-nm range, after correction, it results in $\langle\alpha(\mathrm{I})\rangle$ $=0.93 \pm 0.07$.

The preceding $\langle\alpha\rangle$ values were confirmed by using $G\left(L_{\mathrm{s}}, t\right)$, the height-to-height correlation function, resulting from the analysis of STM images. ${ }^{1}$ In this case, the following equations were used to evaluate $\alpha$ :

$$
G\left(L_{\mathrm{s}}\right)=\left[1 / N \sum\left[h\left(x_{1}\right)-h\left(x_{0}\right)\right]\right]^{2}
$$

and

$$
G(L) \propto L_{\mathrm{s}}^{2 \alpha}
$$

Again, $\langle\alpha\rangle$ values derived in this way are in good agreement with those obtained by the dynamic scaling (Table 1).

Corrected values of $\langle\alpha\rangle$ resulting at different $T_{\mathrm{s}}$ and solution composition are assembled in Table 1 . These results suggest a change in the surface characteristics of vapor-deposited gold films from fractal to nonfractal, which depends on the mobility of gold atoms at the surface. It is well-known that surface mobility of metal atoms can be enhanced either by increasing $T_{\mathrm{s}}$ or by the presence of adsorbates. ${ }^{15}$ In aqueous $\mathrm{H}_{2} \mathrm{SO}_{4}$ solutions at $323 \mathrm{~K}$, the surface mobility of gold atoms is relatively small, ${ }^{16}$ and accordingly, the surface characteristics of vapor-deposited gold films remain close to those observed before aging the film in the electrolyte solution, at least for the interval of time covered by this work.

On the other hand, the addition of $\mathrm{KCl}$ to aqueous $\mathrm{H}_{2} \mathrm{SO}_{4}$ solutions at $323 \mathrm{~K}$ leads to an increase in the value of $\alpha$. This means that in the KCl-containing solution the gold film surface smoothness is greater than that for those

(15) Bonzel, H. P. In Surface Physics of Materials; Blakely, J. M. Ed.; Academic Press: New York, 1975; p 280.

(16) Trevor, D.; Chidsey, C. E. D.; Loiacono, D. N. Phys. Rev. Lett. 1988, 62, 1384 

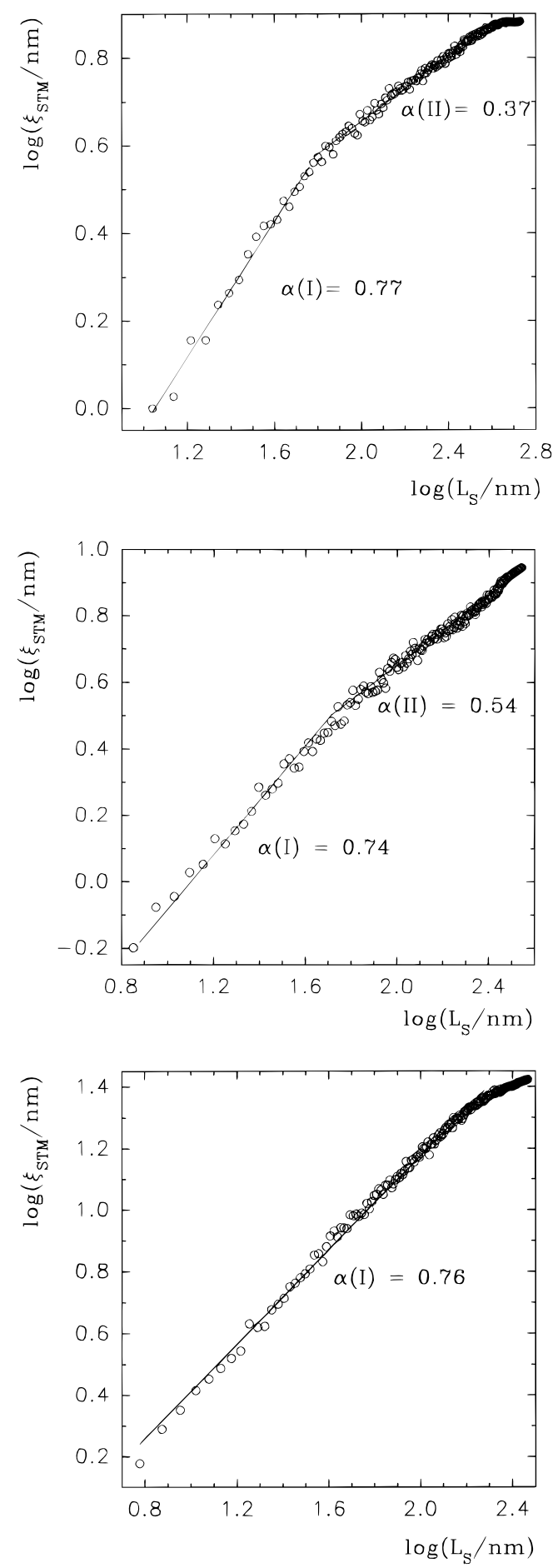

Figure 6. $\log \xi_{\mathrm{STM}} \mathrm{vs} \log L_{\mathrm{s}}$ plot from gold films grown at $v=$ $0.1 \mathrm{~nm} \mathrm{~s}^{-1}$ : (a, top) $T_{\mathrm{s}}=298 \mathrm{~K}$; (b, middle) $T_{\mathrm{s}}=298 \mathrm{~K}$ after the film was kept in contact with $0.5 \mathrm{M} \mathrm{H}_{2} \mathrm{SO}_{4}+0.5 \mathrm{M} \mathrm{KCl}$ for $24 \mathrm{~h}$ at $323 \mathrm{~K}$; (c, bottom) $T_{\mathrm{s}}=673 \mathrm{~K}$.

films without aging, or after aging in plain aqueous $\mathrm{H}_{2} \mathrm{SO}_{4}$ solution. This fact agrees with previously reported observations ${ }^{17}$ that small amounts of $\mathrm{Cl}^{-}$ions in acid solution are able to eliminate surface roughness at metal surfaces. ${ }^{17}$

The values $\alpha(\mathrm{II}) \cong 0.43$ and $\alpha(\mathrm{II}) \cong 0.56$ obtained in $\mathrm{KCl}$-free and $0.5 \mathrm{M} \mathrm{KCl-containing} \mathrm{H}_{2} \mathrm{SO}_{4}$ aqueous solution, respectively, lie between $\alpha$ (II) $\cong 0.4$ and $\alpha \cong 0.90$ obtained at the low and high $T_{\mathrm{s}}$ values. These data suggest that the surface diffusion of gold atoms partially removes surface irregularities for lengths greater than $d_{s}$, depending on the surface mobility induced by anion adsorption. This explanation is basically supported by the increase in $D_{\mathrm{s}}$, the surface diffusion coefficient of gold atoms, by the presence of $\mathrm{Cl}^{-}$ions in the solution. Thus, $D_{\mathrm{s}}$ changes from $10^{-14} \mathrm{~cm}^{2} \mathrm{~s}^{-1}$ in $0.5 \mathrm{M} \mathrm{H}_{2} \mathrm{SO}_{4}$ to $10^{-11} \mathrm{~cm}^{2} \mathrm{~s}^{-1}$ in $\mathrm{H}_{2} \mathrm{SO}_{4}+0.05 \mathrm{M} \mathrm{KCl}^{16}$

Values of $\alpha$ (II) shown in Table 1 indicate a continuous variation of $\alpha$ describing the roughness decay which leads to a faceted surface with $\alpha=0.90$. However, for $t \Longrightarrow \infty$, the enhanced gold surface atom mobility assisted either by increasing $T_{\mathrm{s}}$ or by anion adsorption would tend to produce a completely smooth surface $(\alpha=0)$. Thus, the observed values of $\alpha(\mathrm{II})$ and $\alpha(\mathrm{I}) \cong 0.90$ would reflect transient regimes of the surface, and therefore, a crossover to the Edwards-Wilkinson scaling ${ }^{18}(\alpha=0)$ would be expected.

\section{Conclusions}

Results shown in this work provide a quantitative approach to the role of gold surface atom diffusion in the change of fractal properties of gold film surfaces. Accordingly, the disordered structure produced by vapor deposition at $T_{\mathrm{s}}=298 \mathrm{~K}$ and characterized by $\alpha \cong 0.4$ tends to disappear either by the presence of adsorbable anions from the electrolyte solution or by increasing the substrate temperature leading to better-ordered surface structures. The extent of surface rearrangement in aqueous solutions depends on the strength of anion adsorption and anion concentration in the electrolyte.

Acknowledgment. This work was financially supported by the Consejo Nacional de Investigaciones Cientificas y Técnicas of Argentina (CONICET) and by the scientific cooperation agreement between CONICET and CSIC, Consejo Superior de Investigaciones Científicas of Spain. M.E.V. is a member of the Research Career of CIC (Bs As).

\section{LA9406923}

(17) García, P.; Gómez, M. M.; Salvarezza, R. C.; Arvia, A. J. J. Electroanal. Chem. 1993, 347, 237.

(18) Edwards, S. F.; Wilkinson, D. R. Proc. R. Soc. London, A 1982, 381,17 . 